\title{
Inguinal hernia: why is it still a topic of debate?
}

\author{
G. Campanelli ${ }^{1}$
}

Published online: 25 September 2021

(c) The Author(s), under exclusive licence to Springer-Verlag France SAS, part of Springer Nature 2021

Inguinal hernia repair alone accounts for over $80 \%$ of all abdominal wall surgeries. Being the most common surgical procedure, performed in operating theaters the world over, its importance-epidemiological, social, economic and scientific-is clearly considerable.

Since the practice of inguinal hernia repair surgery is unrelated to a country's economic development, its diffusion is global. Performed on a daily basis, it is a procedure that, in routine cases, should be within the capabilities of all surgeons, even young and relatively inexperienced ones.

To fill any gaps in training that may exist, international scientific societies, and also numerous prominent national ones, have set up schools aimed at young (and not so young) surgeons. The aim of these schools is to ensure that the outcomes of surgical techniques no longer depend on the level of training received by the operator, but instead reflect the true value of the techniques themselves.

At the same time, scientific societies are continually developing guidelines that are supported, to different degrees, by real and incontrovertible evidence.

With all this in mind, then, it would seem that, in the case of primary inguinal hernia at least, we are reaching a point at which many doubts can be dispelled, making it acceptable to establish a sort of uniformity of indications and treatment.

While this is true in theory, it is also true that the different techniques continuously introduced and reintroduced internationally, like the countless devices now available, have the effect of generating a variegated array of opinions, attitudes and surgical choices, as well as plenty of studies of other, more or less important, aspects of inguinal hernia surgery.

In this issue of HERNIA, we have gathered together a series of valid scientific articles-all have passed, as usual, the scrutiny of rigorous blinded peer review processeswith the precise aim of hearing and disseminating opinions, thoughts, and above all different experiences in this area.

HERNIA, as you know, aims, among other things, to stimulate valuable scientific debate among its authors and readers, and the progressive increase in Letters and Comments we receive bears this out. In this spirit, we will be delighted to receive pertinent comments on this issue, too.

\section{Declarations}

Conflict of interest The authors declare that they have no conflict of interest.

Ethical approval This article does not contain any studies with human participants or animals performed by any of the authors.

Informed consent For this type of study formal consent is not required.

Publisher's Note Springer Nature remains neutral with regard to jurisdictional claims in published maps and institutional affiliations.

G. Campanelli

hernia.editorialoffice@gmail.com

1 University of Insubria, Gruppo Ospedaliero San Donato, Milan, Italy 